



\title{
Revista de Ciencias Sociales
}

\section{Universidad de Costa Rica}

Número 140 (II), 2013, Edición trimestral

\author{
ISSN 0482-5276 \\ DIRECTOR Daniel Camacho Monge \\ REVISTA.cs@ucr.ac.cr \\ EDITORA María Fernanda Arguedas Abarca \\ mariafernanda.arguedas@ucr.ac.cr \\ ASISTENTES DE EDICIÓN Ana María Acosta Barrientos \\ Flavio Mora Moraga \\ INFORMACIÓN http://revistacienciassociales.ucr.ac.cr \\ Teléfono: (506) 2511-8703 \\ CORRESPONDENCIA Apartado 49-2060 \\ Ciudad Universitaria Rodrigo Facio \\ Costa Rica, América Central \\ REVISTA.cs@ucr.ac.cr \\ CANJES Sistema de Bibliotecas, Documentación e Información \\ Unidad de Selección y Adquisiciones-Canje \\ Ciudad Universitaria Rodrigo Facio, Costa Rica \\ canje@sibdi.bld.ucr.ac.cr \\ SUSCRIPCIONES Editorial Universidad de Costa Rica \\ Apartado Postal 11501 \\ 2060 Ciudad Universitaria Rodrigo Facio \\ distribucionyventas.siedin@ucr.ac.cr \\ VALOR DE LA SUSCRIPCIÓN C Costa Rica $\$ 1500$ cada ejemplar. Anual $\$ 6000$ \\ América Latina, Asia y África $\$ 20.00$. Resto del mundo $\$ 80.00$ \\ ESTA REVISTA FUE FUNDADA EN 1956. \\ 2008. Premio Juan Carlos Portantiero, otorgado por el \\ Consejo Latinoamericano de Ciencias Sociales, CLACSO. \\ (C) 2013 \\ Prohibida la reproducción total o parcial. \\ EDITORIAL Todos los derechos reservados. \\ UCR Hecho el depósito de ley
}

Algunos derechos reservados bajo Licencia

Creative Commons Attribution-NonCommercial-NoDerivs 3.0 Costa Rica. 



\section{Revista de Ciencias Sociales de la Universidad de Costa Rica}

CONSEJO EDITORIAL

\author{
Ana Cecilia Escalante \\ Omar Hernández \\ Erick Hess \\ Henning Jensen \\ María Pérez \\ Gilbert Vargas
}

\author{
Escuela de Sociología \\ Escuela de Antropología \\ Escuela de Ciencias Políticas \\ Escuela de Psicología \\ Escuela de Ciencias de la Comunicación Colectiva \\ Escuela de Geografía
}

\section{COMITÉ CONSULTIVO INTERNACIONAL}

\author{
Raúl Benítez Manaut \\ Leticia Calvario Martínez \\ Ricardo Córdova Macías \\ Olivier Dabène \\ Fernando Díaz Orueta \\ Francisco Hidalgo Flor \\ Ana Isla \\ María Luisa Lourés Seoane \\ Geoffrey Pleyers \\ Carlos Rafael Rea Rodríguez \\ Marcos Roitman Rosemnann \\ Gerardo Tunal Santiago
}

Instituto de Investigaciones Sociales, Universidad Nacional Autónoma de México, UNAM

Centro de Estudios para el Adelanto de las Mujeres y la Equidad de Género de la H. Cámara de Diputados, México

Fundación Guillermo Ungo, El Salvador Instituto de Estudios Políticos de París (Sciences Po)

Universidad de la Rioja, España

Universidad Central del Ecuador

Universidad de Toronto, Canadá

Universidad Complutense de Madrid

CADIS, Centro de Análisis

y de Intervención Sociológica, EHESS, París

Universidad Autónoma de Nayarit, México

Universidad Complutense de Madrid

Universidad Autónoma Metropolitana, UAM. México

La Revista de Ciencias Sociales de la Universidad de Costa Rica es una publicación trimestral. Difunde los resultados de trabajos científicos de investigación en la ciencia social, producidos por esta universidad en las múltiples disciplinas de esa área. Algunas de estas son: Antropología, Sociología, Psicología, Ciencias Políticas, Ciencias de la Comunicación, Economía, Historia, Educación, Trabajo Social, Geografía, etc. Publica también trabajos de investigadores nacionales e internacionales externos a la Universidad de Costa Rica, que acaten los lineamientos de la revista y sean efectivos aportes a la ciencia social. Esta es una revista temática y como tal, desarrolla uno o varios temas en cada número, a partir de las disciplinas señaladas.

La Revista se dirige a profesionales, investigadores, profesores y estudiantes de las diversas ramas de las Ciencias Sociales y es accesible para un público general con formación media.

Cuenta con un Consejo Editorial multidisciplinario y con un Comité Consultivo Internacional dentro de las Ciencias Sociales. Además con la colaboración de un amplio grupo, también interdisciplinario, de especialistas que dictaminan las propuestas de artículos.

Recibe apoyo de varias dependencias de la Universidad: la Vicerrectoría de Investigación, el Sistema Editorial de Difusión Científica de la Investigación (SIEDIN) y el Instituto de Investigaciones Sociales.

Aparece indizada en diversas fuentes de información internacionales. 
Portada: Sin título. Andrea Arias Rodríguez.

Técnica: Óleo sobre lienzo.

\section{INDIZACIÓN EN FUENTES DE INFORMACIÓN NACIONALES E INTERNACIONALES}

Red de Revistas de Ciencias Sociales en América Latina (Revistalas). Asociación Latinoamericana de Sociología.

Comunidad Universitaria de Unidades de Información especializadas en Ciencias Sociales,

Universidad de Costa Rica http://cuuics.fcs.ucr.ac.cr

Red de Revistas Científicas de América Latina y el Caribe, RedALyC.

Sociological Abstracts Databases. Cambridge Scientific Abstracts, United States.

Gale Digital Archives.

Latindex - Directorio de publicaciones seriadas científicas de América Latina y el Caribe.

CLASE (Citas Latinoamericanas en Ciencias Sociales y Humanidades). UNAM, México D.F.

Censo de Revistas Culturales Iberoamericanas 2000. Asociación de Revistas Culturales de

España, ARCE.

Latin American Studies. Volumen 1. Baltimore, United States.

Latin American Studies. Volumen 2. Baltimore, United States.

Ulrich's on disc. New Jersey, United States.

International Labour Documentation. Switzerland.

Pais International in Print. New York, United States.

Religion Index One: Periodicals. Illinois, United States.

Religion Index Two: Multi-Author Works. Illinois, United States.

Dokumentationsdienst Lateinamerika. Hamburg, Germany.

Historical Abstracts. Part A: Modern History Abstracts. Santa Barbara, C.A.

Institut de l'Information Scientifique et Technique (INIST-CNRS, France).

HAPI Hispanic American periodicals Index, Los Ángeles, UCLA Latin American Center.

NISC. Pennsylvania Co. (Family Studies Database).

Russian Academy of Sciences Bibliographies.

CEDOR Centro Documentazione Oscar Romero, Italia.

Revista

305

R Revista de Ciencias Sociales / Universidad de Costa Rica.--

Vol. 1 (1959)--. -- San José, C. R.: Editorial Universidad de Costa Rica, 1959--

ISSN: $0482-5276$

1. Ciencias Sociales -- Publicaciones periódicas.

2. Publicaciones periódicas costarricenses.

BUCR 


\section{Revista de \\ Ciencias Sociales \\ UNIVERSIDAD DE COSTA RICA}

ISSN: 0482-5276

$\mathrm{N}^{\circ} .140$ (II), 2013

\section{ENFOQUE DE GÉNERO Y CAMBIOS CULTURALES}

\section{GENDER PERSPECTIVE AND CULTURAL CHANGES}

Daniel Camacho Monge

Nancy Piedra Guillén

Roxana Morales Bonilla

Andrés Castillo Vargas

Ivannia Chinchilla Badilla
PRESENTACIÓN

La importancia del enfoque de género en la investigación socio-histórica

The importance of gender in socio-historical research

Movimientos, caminos y rutas de las mujeres

en espacios socioculturales

Movements and pathways of women in socio-cultural spaces

El ejercicio de la violencia simbólica en la atención

en salud de mujeres en edad mediana que experimentan la menopausia: principales resultados de una encuesta CAP

The exercise of symbolic violence in health care of middle-age women experiencing menopause: key results of a KAP survey

Prevención del delito y construcción de feminidades juveniles

Crime prevention and youthful femininity construction

Marina Medan

\section{SISTEMAS POLÍTICOS EN SUDAMÉRICA}

POLITICAL SYSTEMS IN SUDAMERICA

César Ulloa Tapia

Nicolás Falomir Lockhart
Región Andina: sistemas de partidos en análisis Andean region: partys' systems in analysis

La identidad de Unasur: ¿regionalismo post-neoliberal o post-hegemónico? regionalism? 


\section{ARTÍCULOS}

Julieta María Capdevielle El concepto de lucha en la sociología de Bourdieu

$111-124$

María Laura Freyre

The concept of struggle in Bourdieu's sociology

Luis Francisco Vargas Madriz

Psicoterapia Funcional Analítica: descripción general

$125-135$

Ronald Ramírez Henderson

de un modelo centrado en el contexto terapeútico

Functional Analytic Psychotherapy: overview

of a model centered on the therapeutic context

Doris Fernández Carvajal Pesca artesanal y pobreza en comunidades aledañas

$137-152$

al Golfo de Nicoya

Artisanal fishing and poverty in the communities near the Nicoya's Gulf

Chester Urbina Gaitán

Las exposiciones nacionales de Costa Rica 1917-1918

$153-161$

Costa Rican national exhibitions 1917-1918

Viriam Leiva Díaz

Conocimiento de las personas adultas sobre el

$163-173$

Carolina Arguedas Medino

climaterio, andropausia y la sexualidad

Marilyn Hidalgo Castillo

Knowledge of adults about the climacteric, andropause and sexuality

Colaboradores

$175-178$

Próximo número 141 


\section{ENFOQUE DE GÉNERO Y CAMBIOS CULTURALES}

\section{GENDER PERSPECTIVE AND CULTURAL CHANGES}

Daniel Camacho Monge

Director

\section{PRESENTACIÓN}

Los movimientos en pro de la equidad de género constituyen una de las transformaciones culturales más amplia y profunda desde la segunda mitad del siglo Xx. Han conmovido la concepción que la sociedad global tiene de sí misma y han provocado cambios que solo hace 75 años eran inimaginables.

Esto ha tenido su manifestación en el campo de las ciencias sociales, hasta el punto que se ha llegado a hablar de un paradigma científico novedoso, de una epistemología centrada en el enfoque de género. Ejemplo de ello son los artículos que hemos incluido en la sección central de este número denominada ENFOQUE DE GÉNERO Y CAMBIOS CULTURALES. En este sentido, contamos con la colaboración de Piedra, quien realiza un estudio teórico-metodológico para la reflexión y el debate de la participación de las mujeres en el contexto socio-histórico, con la finalidad de visibilizar la condición y el aporte de estas en los procesos históricos, es decir, luchar por una historia inclusiva.

Otro de los estudios en este número, es el presentado por Morales, quien a través de un proyecto presentado en la Universidad Estatal a Distancia (UNED, Costa Rica), realiza una revisión de los aportes de las mujeres en las organizaciones sociales desde tres ejes: danza, defensa de la naturaleza y comités patrióticos. Se realiza una reflexión desde los aportes socioculturales de estas mujeres, así como, los puntos de integración con otros sectores de la sociedad.

Asimismo, Castillo y Chinchilla en su artículo analizan la violencia simbólica que sufren las mujeres en edad mediana que se encuentran en el proceso de menopausia, a través del personal de salud que las atienden en los centros médicos. Este tipo de violencia se presenta muchas veces por la falta de información, conocimientos y concepción que se tiene en torno a esta etapa de vida, por lo cual hacen una propuesta para incentivar y ampliar los conocimientos género-sensitivos del personal de salud, así como una ruptura del paradigma que se tiene actualmente de este proceso.

Medan presenta un trabajo desde Buenos Aires, Argentina, en el cual se describe la construcción de las mujeres como beneficiarias en 
programas de prevención del delito juvenil, los cuales han sido destinados e implementados especialmente, para hombres jóvenes. Por otra parte, permite ciertas apreciaciones importantes sobre los conceptos de personas en riesgo y proyectos de vida.

En nuestro subtema titulado SISTEMAS POLÍTICOS EN SUDAMÉRICA, contamos con la colaboración de Ulloa, quien brinda una explicación de las razones por las cuales colapsó el sistema de partidos en los países de Bolivia, Ecuador y Venezuela. Además, realiza un análisis comparativo de las similitudes y diferencias en los procesos políticos de estos países, así como, los posibles escenarios en los que se podrían enfrentar.

Por otro lado, Falomir realiza un aporte en el caso del proceso de integración regional sudamericano (UNASUR), el cual a través del tiempo ha desbordado una serie de explicaciones y caracterizaciones que han llevado al debate y a la reflexión. De igual manera, se exponen dos conceptos relevantes: regionalismo post-neoliberal y regionalismo post-hegemónico, con el fin de lograr un mejor entendimiento de este acontecimiento.

La sección de ARTículos está conformaba por diversos aportes en diferentes temáticas. Entre ellos, se encuentra el de las autoras Capdevielle y Freyre, quienes realizan un razonamiento sobre el concepto de lucha de clase en la sociología de Bourdieu, para lo cual plantean una distinción entre la lucha en su forma competitiva y la lucha en sentido revolucionario. Además, se apoyan en el concepto de Estrategias de Reproducción Social y en investigaciones empíricas para ejemplificar los planos en que se da la lucha competitiva. Otro aspecto importante es la dimensión simbólica que se le da, en este trabajo, a la lucha de clases, con la finalidad de brindar una mejor comprensión de este concepto.

En el área de Psicología, Vargas y Ramírez exponen las bases teóricas, las características, las conductas y las reglas de intervención referente a la Psicoterapia Funcional Analítica en el contexto terapéutico, la cual se basa en los principios del conductismo radical y del contextualismo funcional. Realizan una descripción detallada de este tipo de intervención, siendo una alternativa diferente a los modelos clásicos. Asimismo, se dirige a los individuos que presentan dificultades para crear y mantener relaciones íntimas o con problemas interpersonales constantes $y$ difusos.

Por otro lado, Fernández nos describe la situación y las vivencias con las que se enfrentan los pescadores artesanales en las comunidades de Morales, Costa de Pájaros y Manzanillo de Chomes (Puntarenas, Costa Rica). Aunado a ello, explica la situación actual de la pesca, el papel del Estado y de las instituciones, el modo de subsistencia de las familias y el apoyo económico que reciben. 
En el área de la Historia Nacional, contamos con un breve aporte de Urbina, quien realiza un recuento de las Exposiciones Nacionales de 1917-1918, en Costa Rica. Asimismo, analiza como fueron utilizados tales eventos por el régimen de Federico Tinoco para crear una imagen política positiva del país, presentándolo como un lugar pacífico y educado.

Finalmente, se presenta la colaboración de las autoras Leiva, Arguedas, Hidalgo y Navarro, en la cual se exhiben y analizan los resultados de un proyecto de investigación sobre el conocimiento que poseen las personas adultas sobre los temas del climaterio y la sexualidad, llegando a demostrarse el concepto erróneo que poseen muchas de ellas y la forma en que es concebido este proceso en sus vida.

Con estas diversas colaboraciones, les presentamos el número 140 a nuestros lectores.

Ciudad Universitaria Rodrigo Facio

Junio, 2013 
